\title{
Multidimensional Signal Recovery in Discrete Evolution Systems via Spatiotemporal Trade Off
}

\author{
Roza Aceska \\ Department of Mathematical Sciences, Ball State University \\ Muncie, IN, USA \\ e-mail address:raceska@bsu.edu \\ Armenak Petrosyan \\ Department of Mathematics, Vanderbilt University \\ Nashville, TN, 37240, USA \\ e-mail address: armenak.petrosyan@vanderbilt.edu \\ Sui Tang \\ Department of Mathematics, Vanderbilt University \\ Nashville, TN, 37240, USA \\ e-mail address: sui.tang@vanderbilt.edu
}

\begin{abstract}
The problem of recovering an evolving signal from a set of samples taken at different time instances, has been well-studied for one-variable signals modeled by $\ell^{2}\left(\mathbb{Z}_{d}\right)$ and $\ell^{2}(\mathbb{Z})$. However, most observed time-variant signals in applications are described by at least two spatial variables. In this paper, we study the spatiotemporal sampling pattern to recover the initial signals modeled by $\ell^{2}\left(\mathbb{Z}_{d_{1}} \times \mathbb{Z}_{d_{2}}\right)$ and $\ell^{2}(\mathbb{Z} \times \mathbb{Z})$ which are evolving in a discrete evolution system and provide specific reconstruction results.
\end{abstract}

Key words and phrases : Distributed sampling, reconstruction, frames.

2010 AMS Mathematics Subject Classification-94A20, 94A12, 42C15, 15 A29.

\section{Introduction}

The ongoing development $[2,3,5,9,10,20]$ in sampling theory suggests to combine coarse spatial samples of a signal's initial state with its later-time samples. In these cases, time-dependency among samples permits a reduction of the number of used expensive sensors via the increase of their usage frequency. The reconstruction of the initial distribution of a signal is achieved by the use of the evolutionary nature of that signal under some certain constraints, which is not fully considered in classical sampling problems $[4,6,7,11,13,15,17,18,19]$. The so-called dynamical sampling problem (motivated by $[14,16]$ ) has been wellstudied in the one-variable setting $[5,9,10,2,3]$ but there have been no results in the multivariable setting. In industrial applications (sampling of air pollution, wireless networks) the observed time-variant signals are described by at least two 
variables. In this paper, we formulate the problem of spatiotemporal sampling for two-variable data and provide specific reconstruction results.

1.1. Stating the Dynamical Sampling Problem. In real-life situations, physical systems evolve over time, under the influence of a family of operators $\left\{A_{t}\right\}_{t>0}$. Let $f_{0}$ be the initial state defined on a domain D. Dynamical sampling problem asks when we can recover the initial state $f_{0}$ by the spatiotemporal sampling data $\left\{S_{X_{t_{i}}} A_{t_{i}} f_{0}: i=1, \ldots, N-1\right\}$, where $S_{X_{t_{i}}}$ is a subsampling operator defined by a coarse sampling set $X_{i} \subset D$ at time instances $t_{i}, i=0, \ldots, N-1$. In other words, we would like to compensate for the lack of sufficient samples of the initial state by adding coarse samples of the evolved states $\left\{A_{t_{i}} f_{0}=f_{t_{i}}, i=0, \ldots, N-1\right\}$. In this way, we can use fewer sampling devices to save budget but lose no information.

The dynamical sampling problem is solved when conditions on the sampling sets and time instances $t_{i}$ are found, such that recovery of the signal is possible, preferably in a stable way. That is, if one (or both) of the following properties is satisfied:

ISP Invertibility sampling property. The operators $A_{t_{0}}, \ldots, A_{t_{N-1}}$, the sampling sets $X_{t_{0}}, \ldots, X_{t_{N-1}}$ and the number of repeated sampling procedures satisfy this condition within a class of signals, if any signal $h$ in that class is uniquely determined by its samples data set.

SSP Stability sampling property. The operators, the sampling sets and the number of repeated samplings satisfy this condition in a fixed class of signals, if for any two signals $h, h_{1}$ in that class, the two norms

$$
\left\|h-h_{1}\right\|_{2}^{2} \text { and } \sum_{i=0}^{N}\left\|S_{X_{t_{i}}} A_{t_{i}}\left(h-h_{1}\right)\right\|_{\ell^{2}}^{2} \text { are equivalent. }
$$

SSP is clearly a stronger property and implies ISP. In $[2,3,9,10]$ the authors have studied the dynamical sampling problem for the discrete spatially invariant evolution system, in which the initial state $f$ is defined on the domain $D=$ $\mathbb{Z}_{d}$ or $\mathbb{Z}$ under certain constraints. At time instance $t=n \in \mathbb{N}$, the initial state $f$ is altered by convolution with a filter $a n$ times to be $A_{n}(f)=a * a * \ldots * a * f=a^{n} f$. At each time instance $t=n$, the altered state $A_{n}(f)$ is under-sampled at a uniform subsampling rate $m$. The invertibility and stability questions have been fully answered under the specific constraints. Namely, for a uniform discrete sampling grid $X=m D \subset D$, specific conditions on $a$ and $N$ are stated so that a function $f$ can be recovered from the samples

$$
\left\{f(X), a * f(X), \cdots,\left(a^{N-1} * f\right)(X)\right\}, \text { for } X \subset D .
$$

The multidimensional dynamical sampling problem we consider in this paper has similarities with problems considered by some other authors. For example, in [12], the authors work in a multivariable shift-invariant space(MSIS) setting, and study linear systems $\left\{L_{j}: j=1, \cdots, s\right\}$ such that one can recover any $f$ in MSIS by uniformly downsampling the functions $\left\{\left(L_{j} f\right): j=1, \cdots, s\right\}$, i.e. taking the generalized samples $\left\{\left(L_{j} f\right)(M \alpha)\right\}_{\alpha \in \mathbb{Z}_{d}, j=1, \cdots, s}$. In dynamical 
sampling, there is only one convolution operator A, and it is applied iteratively to the function $f$. This iterative structure is important for our analysis of the kernel of the arising matrix, and using that special structure we are able to add extra samples outside of the initial uniform sampling greed and get full recovery of the signal.

In addition, certain singularity problems, which can occur due to the specific properties of $a$ when sampling on a uniform grid $X$, have been successfully overcome in the cited papers by adding additional samples. Since most real-life phenomena are described by functions of multiple variables, we find it important to expand the dynamical sampling concept on two variable setting, i.e. $D=$ $\mathbb{Z}_{d_{1}} \times \mathbb{Z}_{d_{2}}$ and $\mathbb{Z} \times \mathbb{Z}$. As we will see later, the two variable problem is more complicated in structure and we find it more subtle to overcome the singularity problems. Studying the stated problem in 3 (and higher) variable setting would require similar coping techniques to the ones we use in this paper to expand the domain from one to two dimensions.

\section{Dynamical sampling on $\mathbb{Z}_{d_{1}} \times \mathbb{Z}_{d_{2}}$}

For a positive integer $d, \mathbb{Z}_{d}$ denotes the finite group of integers modulo $d$. In the finite discrete setting, we work on the domain $D=\mathbb{Z}_{d_{1}} \times \mathbb{Z}_{d_{2}}, d_{1}, d_{2} \in \mathbb{N}^{+}$. Let the operator $A$ act on the signal of interest $f \in \ell^{2}(D)$ as a convolution with some $a \in \ell^{1}(D)$ given by

$$
A f(k, l)=a * f(k, l)=\sum_{(s, p) \in D} a(s, p) f(k-s, l-p), \text { for all }(k, l) \in D .
$$

Note that $A$ is a bounded linear operator that maps $\ell^{2}(D)$ to itself. The initial signal $f$ is evolving in time under the repeated effect of $A$ such that at time instance $t=n$, the evolved signal is $f_{n}=A^{n} f=a * a * \cdots * a * f$ (and $\left.f=f_{0}=A^{0} f\right)$.

We assume that $d_{1}$ and $d_{2}$ are odd numbers, such that $d_{i}=J_{i} m_{i}$ for integers $m_{i} \geq 1, J_{i} \geq 1, i=1,2$. We set the sampling sensors on a uniform coarse grid $X=m_{1} \mathbb{Z}_{d_{1}} \times m_{2} \mathbb{Z}_{d_{2}}$ to sample the initial state $f$ and its temporally evolved states $A f, A^{2} f, \ldots, A^{N-1} f$. Note that, given such a coarse sampling grid, each individual measurement is insufficient for recovery of the sampled state.

Let $S_{X}=S_{m_{1}, m_{2}}$ denote the assigned subsampling operator related to the sampling grid. Specifically,

$$
\left(S_{X} f\right)(k, l)= \begin{cases}f(k, l) & \text { if }(k, l) \in X \\ 0 & \text { otherwise }\end{cases}
$$

For some $N \geq 2$, our objective is to reconstruct $f$ from the combined coarse samples set

$$
\left\{y_{j}=S_{X}\left(A^{j} f\right)\right\}, j=0,1, \ldots, N-1 .
$$

We denote by $\mathcal{F}$ the 2 -dimensional discrete Fourier transform (2d DFT) and use the notation $\hat{x}=\mathcal{F}(x)$. After applying $\mathcal{F}$ to $(2.3)$, due to the two-dimensional 
Poisson's summation formula, we obtain

$$
\hat{y}_{n}(i, j)=\frac{1}{m_{1} m_{2}} \sum_{k=0}^{m_{1}-1} \sum_{l=0}^{m_{2}-1} \hat{a}^{n}\left(i+k J_{1}, j+l J_{2}\right) \hat{f}\left(i+k J_{1}, j+l J_{2}\right)
$$

for $(i, j) \in \mathcal{I}=\left\{0, \cdots, J_{1}-1\right\} \times\left\{0, \cdots, J_{2}-1\right\}$ and $n=0,1, \ldots, N-1$.

Let $\overline{\mathbf{y}}(i, j)=\left(\begin{array}{llll}\hat{y}_{0}(i, j) & \hat{y}_{1}(i, j) & \ldots & \hat{y}_{N-1}(i, j)\end{array}\right)^{T},(i, j) \in \mathcal{I}$ and

$$
\overline{\mathbf{f}}(i, j)=\left(\begin{array}{c}
\hat{f}(i, j) \\
\hat{f}\left(i+J_{1}, j\right) \\
\vdots \\
\hat{f}\left(i+\left(m_{1}-1\right) J_{1}, j\right) \\
\hat{f}\left(i, j+J_{2}\right) \\
\vdots \\
\hat{f}\left(i+\left(m_{1}-1\right) J_{1}, j+J_{2}\right) \\
\vdots \\
\vdots \\
\hat{f}\left(i, j+\left(m_{2}-1\right) J_{2}\right) \\
\vdots \\
\hat{f}\left(i+\left(m_{1}-1\right) J_{1}, j+\left(m_{2}-1\right) J_{2}\right)
\end{array}\right) .
$$

We use the block-matrices

$$
A_{l, m_{1} m_{2}}(i, j)=\left(\begin{array}{cccc}
\hat{a}\left(i, j+l J_{2}\right) & \hat{a}\left(i+J_{1}, j+l J_{2}\right) & \cdots & \hat{a}\left(i+\left(m_{1}-1\right) J_{1}, j+l J_{2}\right) \\
\vdots & \vdots & \vdots & \vdots \\
\hat{a}^{N-1}\left(i, j+l J_{2}\right) & \hat{a}^{N-1}\left(i+J_{1}, j+l J_{2}\right) & \cdots & \hat{a}^{N-1}\left(i+\left(m_{1}-1\right) J_{1}, j+l J_{2}\right)
\end{array}\right),
$$

where $l=0,1, \ldots, m_{2}-1$, to define the $N \times m_{1} m_{2}$ matrix

$$
\mathcal{A}_{m_{1}, m_{2}}(i, j)=\left[A_{0, m_{1} m_{2}}(i, j) A_{1, m_{1} m_{2}}(i, j) \ldots A_{m_{2}-1, m_{1} m_{2}}(i, j)\right]
$$

for all $(i, j) \in \mathcal{I}$. Equations (2.4) have the form of vector inner products, so we restate them in matrix product form

$$
\overline{\mathbf{y}}(i, j)=\frac{1}{m_{1} m_{2}} \mathcal{A}_{m_{1}, m_{2}}(i, j) \overline{\mathbf{f}}(i, j)
$$

By equation (2.6), we need $N \geq m_{1} m_{2}$ to be able to recover the signal $f$. Note that for $N=m_{1} m_{2}$, matrix (2.5) is square, we denote this special square matrix by $A_{m_{1}, m_{2}}(i, j)$ and obtain the following reconstruction result:

Proposition 1. For $N=m_{1} m_{2}$, the SSP is satisfied if and only if

$$
\operatorname{det} A_{m_{1}, m_{2}}(i, j) \neq 0 \text { for all }(i, j) \in \mathcal{I} \text {. }
$$

In the finite dimensional case, unique reconstruction is equivalent to stable reconstruction so SSP and ISP coincide. When (2.7) holds true, the signal is recovered from the system of equations

$$
\overline{\mathbf{f}}(i, j)=m_{1} m_{2} A_{m_{1}, m_{2}}^{-1}(i, j) \overline{\mathbf{y}}(i, j),(i, j) \in \mathcal{I} .
$$


As expected, Proposition 1 reduces to the respective result in [9] when $d=d_{1}$ and $d_{2}=1$, or $d=d_{2}$ and $d_{1}=1$.

2.1. Extra samples for stable spatiotemporal sampling. Proposition 1 gives a complete characterization of stable recovery from the dynamical samples (2.3). In practice, however, we may not have the ideal filter $a$ such that (2.7) holds true. For instance, consider a kernel $a$ with a so-called quadrantal symmetry, i.e. let

$$
\hat{a}(s, p)=\hat{a}\left(d_{1}-s, p\right)=\hat{a}\left(s, d_{2}-p\right)=\hat{a}\left(d_{1}-s, d_{2}-p\right)
$$

for all $(s, p) \in D$. Since (2.5) is a Vandermonde matrix, it is singular if and only if some of its columns coincide. In this case, it is easy to see that $A_{m_{1}, m_{2}}(0,0)$ is singular, which prevents the stable reconstruction.

Motivated by the above example, we propose a way of taking extra samples to overcome the lack of reconstruction uniqueness, whenever singularities for matrix (2.5) occur. Let

$$
\mathcal{A}=\left(\begin{array}{cccc}
A_{m_{1}, m_{2}}(0,0) & 0 & \ldots & 0 \\
0 & A_{m_{1}, m_{2}}(1,0) & \ldots & 0 \\
\vdots & \vdots & \ddots & \vdots \\
0 & 0 & \ldots & A_{m_{1}, m_{2}}\left(J_{1}-1, J_{2}-1\right)
\end{array}\right)
$$

and

$$
\overline{\mathbf{f}}=\left(\begin{array}{c}
\overline{\mathbf{f}}(0,0) \\
\overline{\mathbf{f}}(1,0) \\
\vdots \\
\overline{\mathbf{f}}\left(J_{1}-1,0\right) \\
\overline{\mathbf{f}}(1,1) \\
\vdots \\
\overline{\mathbf{f}}\left(J_{1}-1,1\right) \\
\vdots \\
\vdots \\
\overline{\mathbf{f}}\left(0, J_{2}-1\right) \\
\vdots \\
\overline{\mathbf{f}}\left(J_{1}-1, J_{2}-1\right)
\end{array}\right), \overline{\mathbf{y}}=\left(\begin{array}{c}
\overline{\mathbf{y}}(0,0) \\
\overline{\mathbf{y}}(1,0) \\
\vdots \\
\overline{\mathbf{y}}\left(J_{1}-1,0\right) \\
\overline{\mathbf{y}}(1,1) \\
\vdots \\
\overline{\mathbf{y}}\left(J_{1}-1,1\right) \\
\vdots \\
\vdots \\
\overline{\mathbf{y}}\left(0, J_{2}-1\right) \\
\vdots \\
\overline{\mathbf{y}}\left(J_{1}-1, J_{2}-1\right)
\end{array}\right)
$$

Then

$$
\mathcal{A} \overline{\mathbf{f}}=\overline{\mathbf{y}}
$$

and

$$
\operatorname{ker}(\mathcal{A})=\bigoplus_{(i, j) \in \mathcal{I}} \operatorname{ker}\left[A_{m_{1}, m_{2}}(i, j)\right]
$$

The kernels of each $A_{m_{1}, m_{2}}(i, j)$ can be viewed as generated by linearly independent vectors $\hat{v}_{j} \in \ell^{2}(D)$ such that each $\hat{v}_{j}$ has exactly two nonzero coordinates, one of which is equal to 1 and the other is -1 . Let's assume that the nullity of matrix $A_{m_{1}, m_{2}}(i, j)$ equals $w_{i, j}$ at each $(i, j) \in \mathcal{I}$. Then there are $n=\sum_{i, j} w_{i, j}$ 
of such linearly independent vectors $\hat{v}_{j} \in \ell^{2}(D)$. Let $\left\{v_{j}: j=1, \cdots, n\right\}$ be their image under the $2 D$ inverse DFT. Note that $\left\{v_{j}: j=1, \cdots, n\right\} \subset \ell^{2}(D)$ is also linearly independent.

Let $\Omega \subset D \backslash X$ be the additional sampling set, that is to say, we take extra spatial samples of the initial state $f$ at the locations specified by $\Omega$. By $S_{\Omega}$ we denote the related sampling operator and $R_{\Omega}$ is a $|\Omega| \times n$ matrix with rows corresponding to $\left[v_{1}(k, l), \cdots, v_{n}(k, l)\right]_{\{(k, l) \in \Omega\}}$. With these notations, the following result holds true:

Theorem 2.1. The reconstruction of $f \in \ell^{2}(D)$ from its spatiotemporal samples

$$
\left\{S_{\Omega} f, S_{X} f, S_{X} A f, \cdots, S_{X} A^{m_{1} m_{2}-1} f\right\}
$$

is possible in a stable manner (SSP is satisfied) if and only if $\operatorname{rank}\left(R_{\Omega}\right)=n$.

In particular, if SSP holds true, then we must have $|\Omega| \geq n$.

Proof. Let $W=\operatorname{span}\left\{v_{j}: j=1, \cdots, n\right\}$. It suffices to show that

$$
\operatorname{ker}\left(S_{\Omega}\right) \cap W=\{0\} \text { if and only if } \operatorname{rank}\left(R_{\Omega}\right)=n .
$$

Suppose $w$ is in $\operatorname{ker}\left(S_{\Omega}\right) \cap W$. There must exist coefficients $c_{1}, c_{2}, . ., c_{n}$ so that $w=\sum_{j=1}^{n} c_{j} v_{j}$ and $S_{\Omega} w=0$. The last statement is equivalent to

$$
\left[v_{1}(k, l), v_{2}(k, l), \cdots, v_{n}(k, l)\right]\left[c_{1} c_{2} \ldots c_{n}\right]^{T}=0
$$

for each $(k, l) \in \Omega$. Equivalently, we have $R_{\Omega} \mathbf{c}=0$. Hence, $\mathbf{c}=0$ if and only if $\operatorname{rank}\left(R_{\Omega}\right)=n$.

Since the $d_{1} d_{2} \times n$ matrix $R=\left[v_{1}(k, l), \cdots, v_{n}(k . l)\right]_{\{(k, l) \in D\}}$ has column rank $n$, for any kernel $a$, there exists a minimal choice of $\Omega$, namely $|\Omega|=n$ such that the square matrix $R_{\Omega}$ is invertible. It is hard to give a formula to specify the extra sampling set for every kernel $a \in \ell^{2}(D)$. On the other hand, compared to the 1 -variable case [9], it is more challenging to specify the rank of $R_{\Omega}$ analytically, since the entries of $R_{\Omega}$ will involve the product of sinusoids mixed with exponentials in general.

In [9], the authors studied a typical low pass filter with symmetric properties and gave a choice of a minimal extra sampling set $\Omega$, since symmetry reflects the fact that there is often no preferential direction for physical kernels and monotonicity is a reflection of energy dissipation. Similarly, we consider a kernel $a$ with a so-called strict quadrantal symmetry: for a fixed $(k, l) \in D, \hat{a}(s, p)=$ $\hat{a}(k, l)$ if and only if

$$
(s, p) \in\left\{(k, l),\left(d_{1}-k, l\right),\left(k, d_{2}-l\right),\left(d_{1}-k, d_{2}-l\right)\right\} .
$$

Since $A_{m}(i, j)$ is a Vandermonde matrix, it has singularity if and only if some of its columns coincide. We can compute the singularity of each $A_{m}(i, j)$, as we make use of its special structure.

Lemma 2.2. If the filter a satisfies the symmetry assumptions (2.11), then

$$
\operatorname{dim}(\operatorname{ker}(\mathcal{A}))=\frac{d_{1}\left(m_{2}-1\right)}{2}+\frac{d_{2}\left(m_{1}-1\right)}{2}-\frac{\left(m_{1}-1\right)\left(m_{2}-1\right)}{4} .
$$


Clearly, we need an extra sampling set $\Omega \subset D$ with size $\operatorname{dim}(\operatorname{ker}(\mathcal{A}))$. Based on Theorem 2.1, we provide a minimal $\Omega$ :

Theorem 2.3. Assume that the kernel a satisfies the strict quadrantal symmetry assumptions (2.11) and let

$$
\Omega=\left\{(k, l): k=1 \cdots \frac{m_{1}-1}{2}, l \in \mathbb{Z}_{d_{2}}\right\} \cup\left\{(k, l): k \in \mathbb{Z}_{d_{1}}, l=1, \cdots, \frac{m_{2}-1}{2}\right\} .
$$

Then, any $f \in \ell^{2}(D)$ is recovered in a stable way from the expanded set of samples

$$
\left\{S_{\Omega} f, S_{X} f, S_{X} A f, \cdots, S_{X} A^{m_{1} m_{2}-1} f\right\} .
$$

Remark 2.4. Note that in this case

$$
|\Omega|=\frac{d_{1}\left(m_{2}-1\right)}{2}+\frac{d_{2}\left(m_{1}-1\right)}{2}-\frac{\left(m_{1}-1\right)\left(m_{2}-1\right)}{4},
$$

so by Theorem 2.1 and Lemma 2.2 we can not do better in terms of its cardinality.

Proof. Set

$$
n=\frac{d_{1}\left(m_{2}-1\right)}{2}+\frac{d_{2}\left(m_{1}-1\right)}{2}-\frac{\left(m_{1}-1\right)\left(m_{2}-1\right)}{4} .
$$

Recall that the kernels of singular blocks $A_{m_{1}, m_{2}}(i, j)$ are generated by vectors $\left\{\hat{v}_{k}: k=1, \cdots n\right\}$, such that each $\hat{v}_{k}$ has exactly two non-zero components, 1 and -1 (corresponding to each pair of identical columns). Then the formula of $2 D$ inverse DFT gives

$$
v_{j}(k, l)=\sum_{s=0}^{d_{1}-1} \sum_{p=0}^{d_{2}-1} \hat{v}_{j}(s, p) e^{\frac{2 \pi i s k}{d_{1}}} e^{\frac{2 \pi i p l}{d_{2}}}, \quad(k, l) \in \mathbb{Z}_{d_{1}} \times \mathbb{Z}_{d_{2}} .
$$

We define a row vector $\mathcal{F}_{1}(k)=\left[1, e^{\frac{2 \pi i k}{d_{1}}}, \cdots, e^{\frac{2 \pi i\left(d_{1}-1\right) k}{d_{1}}}\right]$ for all $k \in \mathbb{Z}_{d_{1}}$. For each $l=0,1, \cdots, d_{2}-1$, we define a row vector $\overline{\mathcal{F}}_{2}(l)$ of length $d_{2}-\frac{m_{2}-1}{2}$, which is derived from vector

$$
\left[1, e^{\frac{2 \pi i l}{d_{2}}}, \cdots, e^{\frac{2 \pi i\left(d_{2}-1\right) l}{d_{2}}}\right]
$$

after deleting the entries that correspond to $\left\{s J_{2}+1: 1 \leq s \leq \frac{m_{2}-1}{2}\right\}$, i.e. we omit the entries $e^{\frac{2 \pi s J_{2}}{d_{2}}}$ for $1 \leq s \leq \frac{m_{2}-1}{2}$. We reorder the vectors $v_{j}$ so that $\left[v_{1}(k, l), \cdots, v_{n}(k, l)\right]$ equals

$$
2 i\left[\sin \left(\frac{2 \pi 1 l}{m_{2}}\right) \mathcal{F}_{1}(k), \cdots, \sin \left(\frac{2 \pi\left(m_{2}-1\right) l}{2 m_{2}}\right) \mathcal{F}_{1}(k), \sin \left(\frac{2 \pi 1 k}{m_{1}}\right) \overline{\mathcal{F}}_{2}(l) \cdots, \sin \left(\frac{2 \pi\left(m_{1}-1\right) k}{2 m_{1}}\right) \overline{\mathcal{F}}_{2}(l)\right]
$$

for every $(k, l) \in \Omega$. By Theorem 2.1, the proof is complete if we show that these $n=|\Omega|$ row vectors of size $n$ are linearly independent.

We define a row vector $R(k, l)$ corresponding to $(k, l) \in \Omega$ given by

$$
2 i\left[\sin \left(\frac{2 \pi 1 l}{m_{2}}\right) \mathcal{F}_{1}(k), \cdots, \sin \left(\frac{2 \pi\left(m_{2}-1\right) l}{2 m_{2}}\right) \mathcal{F}_{1}(k), \sin \left(\frac{2 \pi 1 k}{m_{1}}\right) \overline{\mathcal{F}}_{2}(l) \cdots, \sin \left(\frac{2 \pi\left(m_{1}-1\right) k}{2 m_{1}}\right) \overline{\mathcal{F}}_{2}(l)\right] .
$$


Suppose that for some coefficients $\{c(k, l):(k, l) \in \Omega\}$, it holds

$$
\sum_{(k, l) \in \Omega} c(k, l) R(k, l)=0 .
$$

We need to show that all $c(k, l)=0$. Note that, for a fixed $k$, the vector $R(k, l)$ is compartmentalized into two components with lengths $\frac{m_{2}-1}{2}$ and $\frac{m_{1}-1}{2}$. By construction, $\left\{\mathcal{F}_{1}(k) \mid k \in \mathbb{Z}_{d_{1}}\right\}$ are linearly independent row vectors. Then, the coefficients related to $\mathcal{F}_{1}(k)$ for the first component should be zeros. Related to the first component of length $\frac{m_{2}-1}{2}$, for every fixed $k \in \mathbb{Z}_{d_{1}}$ such that $(k, l) \in \Omega$ for some $l$, the following $m_{2}$ equations hold true

$$
\sum_{(k, l) \in \Omega} c(k, l) \sin \left(\frac{2 \pi s l}{m_{2}}\right)=0 \text { for } s=0,1, \ldots, m_{2}-1 .
$$

Case I: if $k \geq \frac{m_{1}+1}{2}$ or $k=0$, then $(k, l) \in \Omega$ if and only if $l=1, \cdots \frac{m_{2}-1}{2}$. We restate the system of equations (2.14) in the matrix form:

$$
\left(\begin{array}{cccc}
\sin \left(\frac{2 \pi}{m_{2}}\right) & \sin \left(\frac{4 \pi}{m_{2}}\right) & \ldots & \sin \left(\frac{\pi\left(m_{2}-1\right)}{m_{2}}\right) \\
\sin \left(\frac{4 \pi}{m_{2}}\right) & \sin \left(\frac{8 \pi}{m_{2}}\right) & \ldots & \sin \left(\frac{2 \pi\left(m_{2}-1\right)}{m_{2}}\right) \\
\vdots & \vdots & \ddots & \vdots \\
\sin \left(\frac{\pi\left(m_{2}-1\right)}{m_{2}}\right) & \sin \left(\frac{2 \pi\left(m_{2}-1\right)}{m_{2}}\right) & \ldots & \sin \left(\frac{\pi\left(m_{2}-1\right)\left(m_{2}-1\right)}{2 m_{2}}\right)
\end{array}\right)\left(\begin{array}{c}
c(k, 1) \\
c(k, 2) \\
\vdots \\
c\left(k, \frac{m_{2}-1}{2}\right)
\end{array}\right)=\mathbf{0} .
$$

The matrix on the left-hand side is invertible, since

$$
\left\{\sin (2 \pi x), \sin (4 \pi x), \ldots, \sin \left(\left(m_{2}-1\right) \pi x\right)\right\}
$$

is a Chebyshev system on $[0,1]($ see[8]); Hence we have $c(k, l)=0$ for $l=1, \cdots \frac{m_{2}-1}{2}$.

Case II: if $1 \leq k \leq \frac{m_{1}-1}{2}$, then $(k, l) \in \Omega$ if and only if $l=0, \cdots, d_{2}-1$. Then (2.14) is equivalent to the system of equations

$$
\sum_{l=0}^{d_{2}-1} c(k, l) \sin \left(\frac{2 \pi s l}{m_{2}}\right)=0 \text { for } s=1,2, \ldots,\left(m_{2}-1\right) / 2 .
$$

Related to the second component of length $\frac{m_{1}-1}{2}$, and combined with the fact that $c(k, l)=0$ if $k$ is in case I, for all $s=1,2, \ldots, \frac{m_{1}-1}{2}$ we have

$$
\sum_{l=0}^{d_{2}-1}\left(\sum_{k=1}^{\frac{m_{1}-1}{2}} c(k, l) \sin \left(\frac{2 \pi s k}{m_{1}}\right) \overline{\mathcal{F}}_{2}(l)\right)=0 .
$$

Let $\overline{\mathcal{F}}_{2}=\left[\overline{\mathcal{F}}_{2}(0)^{T}, \ldots, \overline{\mathcal{F}}_{2}\left(d_{2}-1\right)^{T}\right]$, where $\overline{\mathcal{F}}_{2}(l)^{T}$ denotes the transpose of each row vector $\overline{\mathcal{F}}_{2}(l) ; \overline{\mathcal{F}}_{2}$ is a $\left(d_{2}-\frac{m_{2}-1}{2}\right) \times d_{2}$ matrix. Using matrix 
notation, the first equation in (2.16) can be restated as a product, namely

$$
\overline{\mathcal{F}}_{2} \cdot\left(\begin{array}{c}
\sum_{k=1}^{\frac{m_{1}-1}{2}} \sin \left(\frac{2 \pi k}{m_{1}}\right) c(k, 0) \\
\sum_{k=1}^{\frac{m_{1}-1}{2}} \sin \left(\frac{2 \pi k}{m_{1}}\right) c(k, 1) \\
\vdots \\
\frac{m_{1}-1}{2} \sin \left(\frac{2 \pi k}{m_{1}}\right) c\left(k, d_{2}-1\right)
\end{array}\right)=0
$$

As an easy consequence of equation (2.15), for each $1 \leq j \leq \frac{m_{2}-1}{2}$, it holds

$$
\sum_{k=1}^{\frac{m_{1}-1}{2}} \sin \left(\frac{2 \pi k}{m_{1}}\right) \sum_{l=0}^{d_{2}-1}\left(\sin \left(\frac{2 \pi l j}{m_{2}}\right) c(k, l)\right)=0,
$$

which is equivalent to

$$
\begin{aligned}
& \sum_{k=1}^{\frac{m_{1}-1}{2}} \sum_{l=0}^{d_{2}-1} \sin \left(\frac{2 \pi l j}{m_{2}}\right) \sin \left(\frac{2 \pi k}{m_{1}}\right) c(k, l)=0, \\
& \text { i.e. } \quad \sum_{l=0}^{d_{2}-1} \sin \left(\frac{2 \pi l j}{m_{2}}\right) \sum_{k=1}^{\frac{m_{1}-1}{2}} \sin \left(\frac{2 \pi k}{m_{1}}\right) c(k, l)=0 .
\end{aligned}
$$

We define a $\frac{m_{2}-1}{2} \times d_{2}$ matrix $E$ as follows:

$$
E=\left(\begin{array}{cccc}
\sin \left(\frac{2 \pi \cdot 0}{m_{2}}\right) & \sin \left(\frac{2 \pi \cdot 1}{m_{2}}\right) & \ldots & \sin \left(\frac{2 \pi\left(d_{2}-1\right)}{m_{2}}\right) \\
\sin \left(\frac{4 \pi \cdot 0}{m_{2}}\right) & \sin \left(\frac{4 \pi \cdot 1}{m_{2}}\right) & \ldots & \sin \left(\frac{4 \pi\left(d_{2}-1\right)}{m_{2}}\right) \\
\vdots & \vdots & \ldots & \vdots \\
\sin \left(\frac{\pi\left(m_{2}-1\right) \cdot 0}{m_{2}}\right) & \sin \left(\frac{2 \pi\left(m_{2}-1\right)}{m_{2}}\right) & \ldots & \sin \left(\frac{\pi\left(m_{2}-1\right)\left(d_{2}-1\right)}{m_{2}}\right)
\end{array}\right) .
$$

Due to (2.18), we have

$$
E \cdot\left(\begin{array}{c}
\sum_{k=1}^{\frac{m_{1}-1}{2}} \sin \left(\frac{2 \pi k}{m_{1}}\right) c(k, 0) \\
\sum_{k=1}^{\frac{m_{1}-1}{2}} \sin \left(\frac{2 \pi k}{m_{1}}\right) c(k, 1) \\
\vdots \\
\frac{m_{1}-1}{2} \sin \left(\frac{2 \pi k}{m_{1}}\right) c\left(k, d_{2}-1\right)
\end{array}\right)=\mathbf{0}
$$


Let $\mathcal{F}_{2}=\left(\begin{array}{c}E \\ \overline{\mathcal{F}}_{2}\end{array}\right)$. Then

$$
\mathcal{F}_{2} \cdot\left(\begin{array}{c}
\sum_{\substack{k=1 \\
m_{1}-1}}^{\frac{m_{1}-1}{2}} \sin \left(\frac{2 \pi k}{m_{1}}\right) c(k, 0) \\
\sum_{k=1}^{2} \sin \left(\frac{2 \pi k}{m_{1}}\right) c(k, 1) \\
\vdots \\
\frac{m_{1}-1}{2} \sin \left(\frac{2 \pi k}{m_{1}}\right) c\left(k, d_{2}-1\right)
\end{array}\right)=\mathbf{0} .
$$

Note that the $d_{2} \times d_{2}$ matrix $\mathcal{F}_{2}$ is invertible, since it is the image of a series of elementary matrices acting on the $d_{2} \times d_{2}$ DFT matrix (one row minus another row). Hence we have

$$
\left(\begin{array}{c}
\sum_{\substack{k=1 \\
\frac{m_{1}-1}{2}}}^{\frac{m_{1}-1}{2}} \sin \left(\frac{2 \pi k}{m_{1}}\right) c(k, 0) \\
\sum_{k=1}^{2} \sin \left(\frac{2 \pi k}{m_{1}}\right) c(k, 1) \\
\vdots \\
\frac{m_{1}-1}{2} \sin \left(\frac{\pi\left(m_{1}-1\right) k}{m_{1}}\right) c\left(k, d_{2}-1\right)
\end{array}\right)=\mathbf{0}
$$

After analyzing the rest of the equations in (2.16), we obtain:

$$
\sum_{k=1}^{\frac{m_{1}-1}{2}} \sin \left(\frac{2 \pi j k}{m_{1}}\right) c(k, s)=0 \text { for } j=2, \cdots, \frac{m_{1}-1}{2}, s=0,1, \ldots, d_{2}-1 .
$$

In a similar manner, for each $l=0, \cdots, d_{2}-1$ we obtain the matrix equation

$$
\left(\begin{array}{cccc}
\sin \left(\frac{2 \pi}{m_{1}}\right) & \sin \left(\frac{4 \pi}{m_{2}}\right) & \ldots & \sin \left(\frac{\pi\left(m_{1}-1\right)}{m_{1}}\right) \\
\sin \left(\frac{4 \pi}{m_{1}}\right) & \sin \left(\frac{8 \pi}{m_{2}}\right) & \ldots & \sin \left(\frac{2 \pi\left(m_{1}-1\right)}{m_{1}}\right) \\
\vdots & \vdots & \ddots & \vdots \\
\sin \left(\frac{\pi\left(m_{1}-1\right)}{m_{1}}\right) & \sin \left(\frac{2 \pi\left(m_{1}-1\right)}{m_{1}}\right) & \ldots & \sin \left(\frac{\pi\left(m_{1}-1\right)\left(m_{1}-1\right)}{2 m_{1}}\right)
\end{array}\right)\left(\begin{array}{c}
c(1, l) \\
c(2, l) \\
\vdots \\
c\left(\frac{m_{1}-1}{2}, l\right)
\end{array}\right)=0 .
$$

As the matrix on the left hand side is invertible, we must have $c(k, l)=0$ for $k=1, \cdots, \frac{m_{1}-1}{2}$.

We have demonstrated that $c(k, l)=0$ for all $(k, l) \in \Omega$. Therefore the $n$ row vectors $\{R(k, l)\}_{(k, l) \in \Omega}$ are linearly independent i.e. stability of the signal recovery is achieved. 


\section{Dynamical sampling in $\ell^{2}(\mathbb{Z} \times \mathbb{Z})$}

In this section, we aim to generalize our results to signals of infinite length. Somewhat surprisingly, there is not much difference between the techniques used in these two settings and we feel that we can gloss over a few details in the second part without overburdening the reader.

Let $D=\mathbb{Z} \times \mathbb{Z}$. We study a signal of interest $f \in \ell^{2}(D)$ that evolves over time under the influence of an evolution operator $A$. The operator $A$ is described by a convolution with $a \in \ell^{1}(D)$, namely

$$
A f(p, q)=a * f(p, q)=\sum_{k \in \mathbb{Z}} \sum_{l \in \mathbb{Z}} a(k, l) f(p-k, q-l) \text { at all }(p, q) \in D .
$$

Clearly, $A$ is a bounded linear operator, mapping $\ell^{2}(D)$ to itself. Given integers $m_{1}, m_{2} \geq 1$, we assume $m_{1}$ and $m_{2}$ are odd number. We introduce a coarse sampling grid $X=m_{1} \mathbb{Z} \times m_{2} \mathbb{Z}$. We make use of a uniform sampling operator $S_{X}$, defined by $\left(S_{X} f\right)(k, l)=f\left(m_{1} k, m_{2} l\right)$ for $(k, l) \in D$. The goal is to reconstruct $f$ from the set of coarse samples

$$
\left\{\begin{array}{c}
y_{0}=S_{X} f \\
y_{1}=S_{X} A f \\
\cdots \\
y_{N-1}=S_{X} A^{N-1} f .
\end{array}\right.
$$

Similar to the work done in section 2, we study this problem on the Fourier domain. Due to Poisson's summation formula, we have the Lemma below.

Lemma 3.1. The Fourier transform of each $y_{l}$ in $(3.1)$ at $(\xi, \omega) \in \mathbb{T} \times \mathbb{T}$ is

$$
\hat{y}_{l}(\xi, \omega)=\frac{1}{m_{1} m_{2}} \sum_{j=0}^{m_{2}-1} \sum_{i=0}^{m_{1}-1} \hat{a}^{l}\left(\frac{\xi+i}{m_{1}}, \frac{\omega+j}{m_{2}}\right) \hat{f}\left(\frac{\xi+i}{m_{1}}, \frac{\omega+j}{m_{2}}\right) .
$$

Expression (3.2) allows for a matrix representation of the dynamical sampling problem in the case of uniform subsampling. For $j=0,1, \cdots, m_{2}-1$, we define $N \times m_{1}$ matrices

$$
A_{j, m_{1}, m_{2}}(\xi, \omega)=\left(\hat{a}^{k}\left(\frac{\xi+l}{m_{1}}, \frac{\omega+j}{m_{2}}\right)\right)_{k, l},
$$

where $k=0,1, \cdots, N-1, l=0,1, \cdots, m_{1}-1$ and denote by $\mathcal{A}_{m_{1}, m_{2}}(\xi, \omega)$ the block matrix

$$
\left[A_{0, m_{1}, m_{2}}(\xi, \omega) A_{1, m_{1}, m_{2}}(\xi, \omega) \ldots A_{m_{2}-1, m_{1}, m_{2}}(\xi, \omega)\right] .
$$

Let $\overline{\mathbf{y}}(\xi, \omega)=\left(\hat{y}_{0}(\xi, \omega) \hat{y}_{1}(\xi, \omega) \ldots \hat{y}_{N-1}(\xi, \omega)\right)^{T}$ and 


$$
\overline{\mathbf{f}}(\xi, \omega)=\left(\begin{array}{c}
\hat{f}\left(\frac{\xi}{m_{1}}, \frac{\omega}{m_{2}}\right) \\
\hat{f}\left(\frac{\xi+1}{m_{1}}, \frac{\omega}{m_{2}}\right) \\
\vdots \\
\hat{f}\left(\frac{\xi+m_{1}-1}{m_{1}}, \frac{\omega}{m_{2}}\right) \\
\hat{f}\left(\frac{\xi}{m_{1}}, \frac{\omega+1}{m_{2}}\right) \\
\vdots \\
\hat{f}\left(\frac{\xi+m_{1}-1}{m_{1}}, \frac{\omega+1}{m_{2}}\right) \\
\vdots \\
\vdots \\
\hat{f}\left(\frac{\xi}{m_{1}}, \frac{\omega+m_{2}-1}{m_{2}}\right) \\
\vdots \\
\hat{f}\left(\frac{\xi+m_{1}-1}{m_{1}}, \frac{\omega+m_{2}-1}{m_{2}}\right)
\end{array}\right) .
$$

Due to (3.2), it holds

$$
\overline{\mathbf{y}}(\xi, \omega)=\frac{1}{m_{1} m_{2}} \mathcal{A}_{m_{1}, m_{2}}(\xi, \omega) \overline{\mathbf{f}}(\xi, \omega) .
$$

Proposition 2. ISP is satisfied if and only if $\mathcal{A}_{m_{1}, m_{2}}(\xi, \omega)$ as defined in (3.3) has full column rank $m_{1} m_{2}$ at a.e. $(\xi, \omega) \in \mathbb{T} \times \mathbb{T}$, where $\mathbb{T}=[0,1)$ under addition modulo 1. SSP is satisfied if and only if $A_{m_{1}, m_{2}}(\xi, \omega)$ is full rank for all $(\xi, \omega) \in \mathbb{T} \times \mathbb{T}$.

By Proposition 2, we conclude that $N \geq m_{1} m_{2}$. In particular, if $N=m_{1} m_{2}$, then $\mathcal{A}_{m_{1}, m_{2}}(\xi, \omega)$ is a square matrix, we denote by $A_{m_{1}, m_{2}}(\xi, \omega)$ this square matrix.

Corollary 1. When $N=m_{1} m_{2}$, the invertibility sampling property is equivalent to the condition:

$$
\operatorname{det} A_{m_{1}, m_{2}}(\xi, \omega) \neq 0 \text { for a.e. }(\xi, \omega) \in \mathbb{T} \times \mathbb{T} .
$$

Since $A_{m_{1}, m_{2}}(\xi, \omega)$ has continuous entries, the stable sampling property is equivalent to

$$
\operatorname{det} A_{m_{1}, m_{2}}(\xi, \omega) \neq 0 \text { for all }(\xi, \omega) \in \mathbb{T} \times \mathbb{T} \text {. }
$$

From here on we assume $N=m_{1} m_{2}$. By its structure, $A_{m_{1}, m_{2}}(\xi, \omega)$ is a Vandermonde matrix, thus it is singular at $(\xi, \omega) \in \mathbb{T} \times \mathbb{T}$ if and only if some of its columns coincide. In case $A_{m_{1}, m_{2}}(\xi, \omega)$ is singular, no matter how many times we resample the evolved states $A^{n} f, n>N-1$, on the grid $\Omega_{o}=m_{1} \mathbb{Z} \times m_{2} \mathbb{Z}$, the additional data is not going to add anything new in terms of recovery and stability. In such a case we need to consider adding extra sampling locations to overcome the singularities of $A_{m_{1}, m_{2}}(\xi, \omega)$. 
3.1. Additional sampling locations. If $A_{m_{1}, m_{2}}(\xi, \omega)$ is singular at some $(\xi, \omega)$, then by Corollary 1 the recovery of $f \in \ell^{2}\left(\mathbb{Z}^{2}\right)$ is not stable. To remove the singularities and achieve stable recovery, some extra sampling locations need to be added. The additional sampling locations depend on the positions of the singularities of $A_{m_{1}, m_{2}}(\xi, \omega)$ that we want remove. We propose a quasi-uniform way of constructing the extra sampling locations and give a characterization specifying when the singularity will be removed. Then, we use this method to remove the singularity of a strict quadrantally symmetric convolution operator.

Let the additional sampling set be given by

$$
\Omega=\left\{X+\left(c_{1}, c_{2}\right) \mid\left(c_{1}, c_{2}\right) \in W \subset \mathbb{Z}_{m_{1}} \times \mathbb{Z}_{m_{2}}\right\} .
$$

Let $T_{c_{1}, c_{2}}$ denote the translation operator on $\ell^{2}\left(\mathbb{Z}^{2}\right)$, so that $T_{c_{1}, c_{2}} f(k, l)=f(k+$ $\left.c_{1}, l+c_{2}\right)$ for all $(k, l) \in \mathbb{Z}^{2}$. We employ a shifted sampling operator $S_{X} T_{c_{1}, c_{2}}$ to take extra samples at the initial time instance; this means that our subsampling grid is shifted from $X=m_{1} \mathbb{Z} \times m_{2} \mathbb{Z}$ to $\left(c_{1}, c_{2}\right)+X$ and the extra samples are given as

$$
h_{m_{1}, m_{2}}^{c_{1}, c_{2}}=S_{m_{1}, m_{2}} T_{c_{1}, c_{2}} f,\left(c_{1}, c_{2}\right) \in \Omega
$$

Set

$$
u_{c_{1}, c_{2}}(s, p)=e^{2 \pi i \frac{c_{1} s}{m_{1}}} e^{2 \pi i \frac{c_{2} p}{m_{2}}}
$$

for $(s, p) \in \mathbb{Z}_{m_{1}} \times \mathbb{Z}_{m_{2}}$.

By taking the Fourier transform of the samples on the additional sampling set $\Omega$, we obtain

$$
\hat{h}_{m_{1}, m_{2}}^{c_{1}, c_{2}}(\xi, \omega)=\frac{e^{2 \pi i\left(\frac{c_{1} \xi}{m_{1}}+\frac{c_{2} \omega}{m_{2}}\right)}}{m_{1} m_{2}} \sum_{s=0}^{m_{1}-1} \sum_{p=0}^{m_{2}-1} u_{c_{1}, c_{2}}(s, p) \hat{f}\left(\frac{\xi+s}{m_{1}}, \frac{\omega+p}{m_{2}}\right) .
$$

where

$$
u_{c_{1}, c_{2}}(s, p)=e^{2 \pi i \frac{c_{1} s}{m_{1}}} e^{2 \pi i \frac{c_{2} p}{m_{2}}} .
$$

For each $\left(c_{1}, c_{2}\right) \in W$, we define a row vector

$$
u_{c_{1}, c_{2}}=\left\{u_{c_{1}, c_{2}}(s, p)\right\}_{(s, p) \in X}
$$

with terms arranged in the same order as the terms in vector $\overline{\mathbf{f}}(\xi, \omega)$ in (3.4). We organize the vectors $u_{c_{1}, c_{2}}$ in a matrix $\bar{U}=\left(u_{c_{1}, c_{2}}\right)_{\left(c_{1}, c_{2}\right) \in W}$ and extend the data vector $\overline{\mathbf{y}}(\xi, \omega)$ in (3.5) into a big vector $\mathbf{Y}(\xi, \omega)$ by adding

$$
\left\{e^{2 \pi i \frac{-c_{1} \xi}{m_{1}}} e^{2 \pi i \frac{-c_{2} \omega}{m_{2}}}\left(S_{m_{1}, m_{2}} T_{c_{1}, c_{2}} f\right)^{\wedge}(\xi, \omega)\right\}_{\left(c_{1}, c_{2}\right) \in W} .
$$

Then (3.2) and (3.8) can be combined into the following matrix equation

$$
\mathbf{Y}(\xi, \omega)=\frac{1}{m_{1} m_{2}}\left(\begin{array}{c}
\bar{U} \\
A_{m_{1}, m_{2}}(\xi, \omega)
\end{array}\right) \overline{\mathbf{f}}(\xi, \omega)
$$


Proposition 3. If a left inverse for

$$
\left(\begin{array}{c}
\bar{U} \\
A_{m_{1}, m_{2}}(\xi, \omega)
\end{array}\right)
$$

exists for every $(\xi, \omega) \in \mathbb{T}^{2}$, then the vector $f$ can be uniquely and stably recovered from the combined samples (3.1) and (3.6) via (3.9).

If the following property holds true:

$$
\operatorname{ker}(\bar{U}) \cap \operatorname{ker}\left(A_{m_{1}, m_{2}}(\xi, \omega)\right)=0
$$

for every $(\xi, \omega)$ in $\mathbb{T}^{2}$, we say that $W$ removes the singularities of $A_{m}(\xi, \omega)$; In such a case, the assumption in Proposition 3 is satisfied.

Corollary 2. If $W$ removes the singularities of $A_{m}(\xi, \omega)$ then

$$
|W| \geq \operatorname{dim}\left(\operatorname{ker}\left(A_{m_{1}, m_{2}}(\xi, \omega)\right)\right)
$$

for every $(\xi, \omega)$.

3.2. Strict quadrantal symmetric convolution operator. We consider a filter $a$, such that $\hat{a}$ has the strict quadrantal symmetry property, i.e. $\hat{a}\left(\xi_{1}, \omega_{1}\right)=$ $\hat{a}\left(\xi_{2}, \omega_{2}\right)$ for $\left(\xi_{1}, \omega_{1}\right),\left(\xi_{2}, \omega_{2}\right) \in \mathbb{T} \times \mathbb{T}=\mathbb{T}^{2}$ if and only if one of the following conditions is satisfied:

$$
\begin{array}{ll}
\text { 1. } & \xi_{1}=\xi_{2}, \omega_{1}+\omega_{2}=1 \\
\text { 2. } & \xi_{1}+\xi_{2}=1, \omega_{1}=\omega_{2} \\
\text { 3. } & \xi_{1}+\xi_{2}=1, \omega_{1}+\omega_{2}=1 .
\end{array}
$$

The following result is a direct consequence of the symmetries assumptions listed in conditions $1-3$.

Proposition 4. If $\hat{a}(\xi, \omega)$ has the strict quadrantal symmetry property, then we have $\operatorname{det} A_{m_{1}, m_{2}}(\xi, \omega)=0$ when $\xi=0$ or $\omega=0$. Moreover, the kernel of each $A_{m_{1}, m_{2}}(\xi, \omega)$ is a subspace of the kernel of one of the following four matrices:

$$
A_{m_{1}, m_{2}}(0,0), A_{m_{1}, m_{2}}\left(\frac{1}{2}, 0\right), A_{m_{1}, m_{2}}\left(0, \frac{1}{2}\right), A_{m_{1}, m_{2}}\left(\frac{1}{2}, \frac{1}{2}\right) .
$$

From Proposition 4, for a strict quadrantally symmetric kernel we need to consider only the points $(\xi, \omega) \in\left\{(0,0),\left(0, \frac{1}{2}\right),\left(\frac{1}{2}, 0\right),\left(\frac{1}{2}, \frac{1}{2}\right)\right\}$ and construct the set $W$, such that it removes the singularities of the above four matrices.

Proposition 5. If $\hat{a}$ has the strict quadrantal symmetry property, then

$$
\operatorname{dim}\left(A_{m_{1}, m_{2}}(\xi, \omega)\right)=\frac{\left(m_{1}-1\right) m_{2}}{2}+\frac{m_{2}-1}{2} \frac{m_{1}+1}{2}
$$

for every $(\xi, \omega) \in\left\{(0,0),\left(0, \frac{1}{2}\right),\left(\frac{1}{2}, 0\right),\left(\frac{1}{2}, \frac{1}{2}\right)\right\}$.

Proof. We discuss here in depth only the case $\xi=\omega=\frac{1}{2}$. The proof in the other three cases are analogous to what we present here. Because $A_{m_{1}, m_{2}}\left(\frac{1}{2}, \frac{1}{2}\right)$ is a 
Vandermonde matrix, the rank is equal to the number of its different columns. It is easy to show that

$$
\hat{a}\left(\frac{\frac{1}{2}+s}{m_{1}}, \frac{\frac{1}{2}+p}{m_{2}}\right)=\hat{a}\left(\frac{\frac{1}{2}+k}{m_{1}}, \frac{\frac{1}{2}+l}{m_{2}}\right)
$$

is satisfied if and only if one of the following holds true:

(1) $s=k, p+l=m_{2}-1$

(2) $p=l, s+k=m_{1}-1$

(3) $s+k=m_{1}-1, p+l=m_{2}-1$

using which we can easily compute that

$$
\operatorname{dim}\left(\mathcal{A}_{m_{1}, m_{2}}\left(\frac{1}{2}, \frac{1}{2}\right)\right)=\frac{\left(m_{1}-1\right) m_{2}}{2}+\frac{m_{2}-1}{2} \frac{m_{1}+1}{2}=n .
$$

Let

$$
W=W_{1} \cup W_{2}
$$

where

$$
\begin{gathered}
W_{1}=\left\{1, \cdots \frac{m_{1}-1}{2}\right\} \times\left\{0, \cdots, m_{2}-1\right\}, \\
\left.W_{2}=\left\{0, \cdots, m_{1}-1\right\} \times\left\{1, \cdots, \frac{m_{2}-1}{2}\right\}\right\} .
\end{gathered}
$$

Remark 3.2. When $W$ is defined as in (3.11), we have

$$
|W|=\frac{\left(m_{1}-1\right) m_{2}}{2}+\frac{m_{2}-1}{2} \frac{m_{1}+1}{2} ;
$$

By Corollary 2, $W$ has the minimal possible size.

Theorem 3.3. Let $a \in \ell^{1}(D)$ be the filter such that the evolution operator is given by $A x=a * x$. Suppose â satisfies the strict quadrantal symmetric property defined at the beginning of subsection 3.2. Let $\Omega$ be as in (3.6) with $W$ specified in (3.11). Then, any $f \in \ell^{2}(D)$ can be recovered in a stable way from the expanded set of samples

$$
\left\{S_{\Omega} f, S_{X} f, \cdots, S_{X} A^{m_{1} m_{2}-1} f\right\} .
$$

Proof. It suffices to show that for every $(\xi, \omega) \in \mathbb{T} \times \mathbb{T}$, it holds

$$
\operatorname{ker}(\bar{U}) \cap \operatorname{ker}\left(A_{m_{1}, m_{2}}(\xi, \omega)\right)=0 .
$$

By Proposition 4, we only need to study the kernels of these four matrices

$$
A_{m_{1}, m_{2}}(0,0), A_{m_{1}, m_{2}}\left(\frac{1}{2}, 0\right), A_{m_{1}, m_{2}}\left(0, \frac{1}{2}\right), A_{m_{1}, m_{2}}\left(\frac{1}{2}, \frac{1}{2}\right) .
$$

We discuss here in depth for the case $\xi=\omega=\frac{1}{2} . Z:=\operatorname{ker}\left(A_{m_{1}, m_{2}}\left(\frac{1}{2}, \frac{1}{2}\right)\right)$ is a subspace in $\mathbb{C}^{m_{1} m_{2}}$. By Proposition 5 , the dimension of $Z$ is $n$. Taking advantage of the fact that $A_{m_{1}, m_{2}}\left(\frac{1}{2}, \frac{1}{2}\right)$ is a Vandermonde matrix, we can choose a basis $\left\{v_{j}: j=1, \cdots, n\right\}$ for $Z$, such that each $v_{j}$ has only two nonzero entries 1 and 
-1. Let $\mathbf{v} \in \operatorname{ker}(\bar{U}) \cap Z$, there exists $\mathbf{c}=(c(i))_{i=1, \cdots, n}$ such that $\mathbf{v}=\sum_{i=1}^{n} c(i) v_{i}$. Define a $n \times n$ matrix $R$ with the row corresponds to a fixed $\left(c_{1}, c_{2}\right) \in W$ is

$$
\begin{aligned}
& {\left[\left(e^{\frac{2 \pi i\left(m_{1}-1\right) c_{1}}{m_{1}}}-e^{\frac{2 \pi i 0 c_{1}}{m_{1}}}\right) \mathcal{F}_{2}\left(c_{2}\right), \cdots,\left(e^{\frac{2 \pi i\left(m_{1}+1\right) c_{1}}{2 m_{1}}}-e^{\frac{2 \pi i\left(m_{1}-3\right) c_{1}}{2 m_{1}}}\right) \mathcal{F}_{2}\left(c_{2}\right),\right.} \\
& \left.\left(e^{\frac{2 \pi i\left(m_{2}-1\right) c_{2}}{m_{2}}}-e^{\frac{2 \pi i 0 c_{2}}{m_{2}}}\right) \overline{\mathcal{F}}_{1}\left(c_{1}\right), \cdots,\left(e^{\frac{2 \pi i\left(m_{2}+1\right) c_{2}}{2 m_{2}}}-e^{\frac{2 \pi i\left(m_{2}-3\right) c_{2}}{2 m_{2}}}\right) \overline{\mathcal{F}}_{1}\left(c_{1}\right)\right] .
\end{aligned}
$$

Then

$$
\bar{U} \mathbf{v}=0 \text {, which is equivalent to } R \mathbf{c}=0 \text {. }
$$

By the use the same strategy as in the proof of Theorem 2.3, it can be demonstrated that these $n$ row vectors of $R$ are linearly independent. With slight adaptations of the strategy used so far,we can come to the same conclusion for the other three matrices in (3.14). As a consequence of Proposition 3, stability is achieved.

\section{Conclusion}

In this paper, we seek the spatiotemporal trade off in the two variable discrete spatially invariant evolution system driven by a single convolution filter in both finite and infinite case. We characterize the spectral properties of the filters to specify when we can recover the initial state from the uniform undersampled future states and a way to add extra spatial sampling locations to stably recover the signal when the filters fail the certain constraints. Compared to one variable case, the singularity problems caused by the structure of filters are more complicated and tough to solve. We give the explicit constructions of extra spatial sampling locations to resolve the singularity issue caused by the strict quadrantal symmetric filters. Our results can be adapted to the general multivariable case. Different kinds of symmetry assumptions can be imposed on the filters. The problem of finding the right additional spatiotemporal sampling locations for other types of filters remains open and requires a further study.

\section{ACKNOWLEDGEMENT}

We would like to thank Akram Aldroubi for his helpful discussions and comments. The research of Armenak Petrosyan and Sui Tang are partially supported by NSF Grant DMS-1322099.

\section{References}

[1] R. Aceska, A. Petrosyan and S. Tang, Dynamical sampling of two-dimensional temporallyvarying signals, Proceedings of SampTA ,2015.

[2] R. Aceska, A. Aldroubi, J. Davis and A. Petrosyan, Dynamical Sampling in Shift-Invariant Spaces, AMS Contemporary Mathematics (CONM) book series, 2013.

[3] R. Aceska and S. Tang, Dynamical Sampling in Hybrid Shift Invariant Spaces, AMS Contemporary Mathematics (CONM) book series, 2014.

[4] B. Adcock and A. Hansen, A generalized sampling theorem for stable reconstructions in arbitrary bases, J. Fourier Anal. Appl., 18(4), 685-716, 2012. 
[5] A. Aldroubi, U. Molter, C. Cabrelli and S. Tang, Dynamical Sampling. ArXiv 1409.8333. To appear in Appl. Comput. Harmon. Anal.

[6] A. Aldroubi and M. Unser, Sampling Procedures in Function Spaces and Asymptotic equivalence with Shannon's sampling theory, Numer. Func. Anal. and Opt., 15, 1-21, 1994.

[7] A. Aldroubi and K. Gröchenig, Nonuniform sampling and reconstruction in shift-invariant spaces, SIAM Rev., 43, 585-620, 2001.

[8] Andrei Osipov, Vladimir Rokhlin and Hong Xiao, Prolate Spheroidal Wave Functions of Order Zero: Mathematical Tools for Bandlimited Approximation, Springer Science and Business Media, 2013.

[9] A. Aldroubi, J. Davis and I. Krishtal, Dynamical Sampling: Time Space Trade-off, Appl. Comput. Harmon. Anal., 34(3), 495-503, 2013.

[10] A. Aldroubi, J. Davis and I. Krishtal, Exact Reconstruction of Signals in Evolutionary Systems Via Spatiotemporal Trade-off, J. Fourier Anal. Appl., 21(1), 11-31, 2015.

[11] N. Atreas, Perturbed sampling formulas and local reconstruction in shift invariant spaces, J. Math. Anal. Appl., 377, 841-852, 2011.

[12] A. G. García and G. Pérez-Villalón, Multivariate generalized sampling in shift-invariant spaces and its approximation properties, J. Math. Anal. Appl., 355, 397-413, 2009.

[13] P. Jorgensen and Feng Tian, Discrete reproducing kernel Hilbert spaces: Sampling and distribution of Dirac-masses. ArXiv:1501.02310.

[14] Y. Lu and M. Vetterli, Spatial super-resolution of a diffusion field by temporal oversampling in sensor networks, Proc. IEEE Int. Conf. Acoust., Speech and Signal Process. 2009 (ICASSP 2009), 2249 -2252, 2009.

[15] Z. Nashed and Q. Sun, Sampling and reconstruction of signals in a reproducing kernel subspace of $L^{p}\left(\mathbb{R}^{d}\right)$, J. Funct. Anal., 258, 2422-2452, 2010.

[16] J. Ranieri, A. Chebira, Y. M. Lu and M. Vetterli, Sampling and reconstructing diffusion fields with localized sources, Proc. IEEE Int. Conf. Acoust., Speech and Signal Process. 2011 (ICASSP 2011), 4016-4019, 2011.

[17] W. Sun, Sampling theorems for multivariate shift invariant subspaces, Sampl. Theory Signal Image Process., 4, 73-98, 2005.

[18] Q. Sun, Local reconstruction for sampling in shift-invariant spaces, Adv. Comput. Math., 32, 335-352, 2010.

[19] Q. Sun, Nonuniform average sampling and reconstruction of signals with finite rate of innovation, SIAM J. Math. Anal., 38(5), 1389-1422, 2006.

[20] S. Tang, System Identification in Dynamical Sampling, Preprint. ArXiv:1502.02741v2. 